\title{
Haftpflicht bei Körperschädigungen
}

\section{Peter E. Bleuler}

Dr. med., Facharzt für Chirurgie und Handchirurgie, Rüti, Mitglied FMH

Bleibende Folgen von Körperschädigungen sind durch die Leistungen der Sozialversicherungen oft nicht ausreichend abgedeckt. Handelt es sich um einen fremdverursachten Schaden, kann die Haftpflichtleistung des Schadenverursachers im weiteren Leben des Geschädigten eine entscheidende Rolle spielen. Haftpflichtleistungen erhält man nicht automatisch; sie müssen eingefordert werden. Unterlässt der Geschädigte infolge Nicht-Wissens, Unvermögens oder Resignation die Forderung, so geht er unter Umständen der ihm eigentlich zustehenden Chance verlustig, trotz des erlittenen Schadens weiterhin ein selbständiges und finanziell gesichertes Leben führen zu können. Lässt sich der Geschädigte von der Haftpflichtversicherung des Schädigers mit einem ungünstigen Vergleich abspeisen, verzichtet er möglicherweise auf einen erheblichen Teil der ihm gesetzlich zustehenden Leistungen.

Dies ist das Fazit meiner in der letzten Zeit intensiven Beschäftigung mit Haftpflichtgutachten. Da ich im Gespräch mit Kolleginnen und Kollegen den Eindruck gewann, dass die Thematik uns Ärzten zu wenig vertraut ist, erlaube ich mir, hier auf einige Aspekte kurz einzugehen, nicht zuletzt deshalb, weil dem Arzt, der den geschädigten Patienten behandelt, in diesen Fragen eine Beraterfunktion zukommt.

Alle wesentlichen Aussagen des Artikels wurden mit der Rechtsanwältin Dr. iur. Cristina Schiavi diskutiert, die im Zürcher Anwaltsverband der Fachgruppe Haftpflicht- und Versicherungsrecht vorsteht.

\section{Bedeutung des Haftpflichtrechts}

Das Haftpflichtrecht hat eine grosse Bedeutung, weil die Ansprüche aus der Haftpflicht weit über die Sozialversicherungsansprüche hinausgehen. Wir können dabei drei Bereiche unterscheiden, nämlich den Er-

\section{Responsabilité civile lors de lésions corporelles}

En matière de lésions corporelles infligées par une tierce personne, les prétentions en responsabilité civile vont bien au-delà du droit aux prestations sociales et peuvent, pour la personne concernée, revêtir un caractère essentiel pour surmonter les atteintes subies. Le médecin traitant devrait connaître les principaux aspects de cette problématique afin de ne nuire aux intérêts du patient de manière involontaire lors de l'entretien avec ce dernier. werbsschaden, den Haushaltführungsschaden und den Pflegeschaden.

\section{Erwerbsschaden}

Ein ungelernter Arbeiter kann seine dominante rechte Hand auf Grund eines Unfalles nicht mehr einsetzen. Die SUVA und die IV prüfen den Rentenanspruch. In Übereinstimmung mit der Gesetzgebung müssen sie feststellen, wie viel der Verletzte auf einem hypothe-

Der Beizug eines Anwaltes zur Durchsetzung von Haftpflichtansprüchen ist nicht zwingend, aber zu empfehlen.

tisch bestehenden, ausgeglichenen Arbeitsmarkt noch verdienen könnte. Sie kommen zum Schluss, dass dies (theoretisch) 60\% seines früheren Einkommens ausmacht. Somit erhält er eine 40\%-Rente, d.h. er erhält $80 \%$ von $40 \%$ seines früheren Einkommens. In Wirklichkeit findet er auf dem real existierenden Arbeitsmarkt als Einhändiger keine Stelle. Demgegenüber geht das Haftpflichtrecht von der realen Situation, also vom realen Schaden aus. In unserem Beispiel bedeutet dies, dass der Betroffene die übrigen $60 \%$ (+20\% von $40 \%)$ gegenüber dem Haftpflichtigen fordern kann. Davon kann er leben. Hat hingegen jemand in derselben Situation keine Ansprüche auf Haftpflichtleistungen oder macht er sie nicht geltend, wird er vermutlich trotz der Teilrente der Sozialversicherung unter das Existenzminimum fallen und auf Ergänzungsleistungen angewiesen sein oder sogar fürsorgeabhängig werden.

\section{Haushaltführungsschaden}

Betroffen sein kann nicht nur eine Hausfrau, sondern jede und jeder, der oder die vor Eintritt des Schadens einen Teil seines Haushaltes selber führte. Die Haushalttätigkeit, die er oder sie nun nicht mehr leisten kann, wird im Haftpflichtfall zu ca. Fr. 30.- pro Stunde entschädigt. Die Sozialversicherungen hingegen entschädigen keinen Haushaltführungsschaden. Für eine 30-jährige, teilzeitlich arbeitende Frau, die in den nächsten 50 Jahren keine Haushalttätigkeit mehr verrichten kann, ergibt sich ein Haushaltführungsschaden in Millionenhöhe, welcher bei der Haftpflichtversicherung des Schädigers geltend gemacht werden kann. 


\section{Pflegeschaden}

Man stelle sich einen Schwerverletzen vor, z.B. einen Tetraplegiker, der rund um die Uhr der Pflege bedarf. Die IV oder die Unfallversicherung bezahlen in einem solchen Fall eine sogenannte Hilflosen-Entschädigung. Diese deckt aber die Pflegekosten bei weitem nicht. Die unter Umständen sehr erhebliche Differenz kann bei der Haftpflichtversicherung geltend gemacht werden.

\section{Wie kommt der Geschädigte zu den Haftpflichtleistungen?}

Haftpflichtleistungen erhält der Geschädigte nur dann, wenn er sie einfordert. Oft denken die Geschädigten zunächst gar nicht daran, dass ihnen solche Leistungen zustehen. Der Arbeiter, der auf der Baustelle von einem Gerüst herunterstürzt, weiss sich durch die Sozialversicherungen gedeckt und kommt vielleicht nicht auf die Idee, dass der Arbeitgeber, der die Sicherheitsvorschriften beim Gerüstbau vernachlässigt hat, haftpflichtig sein könnte. Die SUVA wird

\section{Zu Beweiszwecken ist ein schriftliches Aufklä- rungsprotokoll zu erstellen, das der Patient nach dem Gespräch unterschreibt.}

den Verletzten nicht unbedingt darauf aufmerksam machen, da sie ihrem Kunden - dem Arbeitgeber ebenso sehr verpflichtet ist. Der Geschädigte wird oft erst dann auf die Haftpflichtfrage aufmerksam, wenn er Versicherungsleistungen vermisst, die ihm nach seinem Rechtsempfinden zustehen, z.B. die Erstattung der Kosten für die Kinderbetreuung, die in der Zeit, in der die Mutter abends Büros putzt und so zum Familienunterhalt beiträgt, anstelle des Geschädigten von einer Drittperson übernommen werden muss. Vollends unverständlich wird es dann für den Patienten, wenn es um die Abgeltung des Dauerschadens geht und die SUVA bei der Berentung eine theoretische Arbeitsfähigkeit in einer "angepassten Tätigkeit» annimmt, für die der Geschädigte aber keine Stelle findet. Doch dann ist es oft zu spät für die Geltendmachung von Haftpflichtansprüchen, die inzwischen verjährt oder verwirkt sind. Wenn in Fällen von vermutetem Drittverschulden der Arzt seinem Patienten frühzeitig den Rat gibt, die Frage zu prüfen, ob Haftpflichtansprüche gegenüber einem allfälligen Schadensverursacher geltend gemacht werden können, dann hat er seinem Patienten unter Umständen einen sehr grossen Dienst erwiesen.

Der Beizug eines Anwaltes zur Durchsetzung von Haftpflichtansprüchen ist nicht zwingend, aber zu empfehlen, und zwar aus folgenden Gründen:
1. Es muss sichergestellt werden, dass der Anspruch nicht verjährt oder verwirkt. Die entsprechenden Fristen und die notwendigen Massnahmen sind, je nach Situation, unterschiedlich. Der Anwalt weiss im Einzelfall, wie vorzugehen ist, um eine Verjährung oder Verwirkung zu vermeiden. Ist der Anspruch einmal verjährt oder verwirkt, kann definitiv keine Forderung mehr durchgesetzt werden, wie im Falle eines Patienten mit einer Geburtslähmung des Armplexus, der einen Tag nach dem 18. Geburtstag den Haftpflichtanspruch anmelden will, weil der Hausarzt damals den Eltern gesagt habe, im Spital sei ein Fehler gemacht worden, der zu einem Haftpflichtanspruch führt; diesen sollen sie geltend machen, sobald der Sohn 18 geworden sei.

2. Der Laie kennt den Umfang des Schadens, den er geltend machen kann, oft nicht, handelt es sich doch um komplizierte Schadensberechnungen, welche auch die sozialversicherungsrechtlichen Ansprüche berücksichtigen müssen. Falls der Geschädigte ein Angebot einer Haftpflichtversicherung erhält, sollte er es von einem Anwalt überprüfen lassen. Nicht selten wird dem nicht anwaltlich vertretenen Geschädigten bis zu $10 \mathrm{Mal}$ weniger angeboten, als ihm von Rechtes wegen zusteht und mit Hilfe eines Anwaltes - oft sogar aussergerichtlich - auch erreichbar wäre.

3. Falls der Geschädigte kein überzeugendes Angebot der Haftpflichtversicherung des Schädigers erhält und klagen muss, kommt er nicht darum herum, einen Anwalt beizuziehen. Die Chancen, seine Ansprüche durchsetzen zu können, sind dann umso besser, je früher der Anwalt eingeschaltet wurde.

\section{Die Rechtsschutzversicherung}

Der Beizug eines Anwaltes kostet Geld. Das Anwaltshonorar bemisst sich nach dem Zeitaufwand, wobei der Stundenansatz vom Streitwert abhängig ist. Geht es um eine Invalidität mit einer hohen Schadensumme, kann eine langedauernde Auseinandersetzung mit den involvierten Versicherungen ein Anwaltshonorar nach sich ziehen, das ein arbeitsunfähiger Kläger kaum aufzubringen imstande ist. Das vorprozessuale Anwaltshonorar des Geschädigten ist aber Teil des haftpflichtrechtlichen Schadens und muss von der Haftpflichtversicherung des Schädigers bezahlt werden. Wenn der Fall aussergerichtlich gelöst wird, entstehen dem Geschädigten somit keine Kosten. Wenn jedoch das Gericht angerufen wird und der Geschädigte unterliegt, muss er nicht nur seine eigenen, son- 
dern auch die Anwaltskosten des Gegners weitgehend übernehmen; ausserdem muss er die ganzen Gerichtsgebühren bezahlen. Dieses Risiko kann im Voraus nie ganz ausgeschlossen werden, denn es liegt in der Natur der Dinge, dass man nicht immer Recht bekommt, wenn man Recht zu haben glaubt. Da es sich um sehr hohe Summen handeln kann, sind viele Geschädigte nicht in der Lage, ein solches Risiko einzugehen und verzichten auf die Klage, selbst wenn sie überzeugt sind, im Recht zu sein und die Chancen gut stehen. Deshalb ist das Vorhandensein einer Rechtsschutzversicherung oft die Voraussetzung zum Klagen. Das Risiko wird dann von der Rechtsschutzversicherung getragen.

\section{Vorschuss der Gerichtsgebühren}

Gemäss der Schweizerischen Zivilprozessordnung kann das Gericht vom Kläger bzw. Geschädigten eine Vorauszahlung der Gerichtsgebühren (und der gegnerischen Anwaltskosten) verlangen, damit er überhaupt klagen kann. Diese Vorschussforderung ist bei unseren Gerichten Usanz geworden. Dafür kommt, falls vorhanden, die Rechtsschutzversicherung auf. Ein Geschädigter, der keine Rechtsschutzversicherung hat, kann somit in echte Schwierigkeiten geraten bzw. gar nicht in der Lage sein, zu klagen. Der Anwalt wird dann zu einer Teilklage raten oder evtl. eine Prozessfinanzierung empfehlen. Er wird auch prüfen, ob der Geschädigte Anspruch auf unentgeltliche Rechtspflege und unentgeltlichen Rechtsbeistand hat. Kommt der Kläger für die Prozessfinanzierung selber auf, besteht das Risiko, dass er, falls er nicht oder nur teilweise Recht bekommt, die Kosten ganz oder teilweise selber tragen muss.

\section{Besonderheiten der Arzthaftpflicht}

Die Arzthaftpflichtfälle machen einen Teil der Haftpflichtansprüche bei Körperschädigungen aus. In der Fachgruppe Haftpflicht- und Versicherungsrecht des Zürcher Anwaltsverbandes besteht der statistisch bisher nicht belegbare Eindruck, dass Haftpflichtansprüche gegen Ärzte und Spitäler in den letzten Jahren häufiger geworden sind. Im Prinzip gelten die oben gemachten Ausführungen genauso wie für die anderen Haftpflichtfälle auch für die Arzthaftpflicht. Eine Besonderheit besteht insofern, als der Arzt, der den
Geschädigten betreut, sich naturgemäss damit schwer tut, dem Patienten zu Haftpflichtforderungen gegen einen Kollegen zu raten. In schweren und eindeutigen Fällen kann der Verzicht auf Haftpflichtansprüche, wie oben aufgeführt, jedoch gravierende Nachteile für den Geschädigten zur Folge haben. Deshalb sollte der Arzt einen Patienten, der in dieser Lage oft zur Resignation neigt, nicht leichtfertig darin unterstützen, den Weg des geringsten Widerstandes einzuschlagen und auf die Geltendmachung von Haftpflichtforderungen zu verzichten. Andererseits sollte man in Fällen, in denen eine Behandlung zwar nicht zum gewünschten Erfolg oder sogar zu einer Komplikation geführt hat, aber kein Arztfehler vorliegt, den Patienten nicht durch unvorsichtige Äusserungen dazu animieren, seinen Arzt einzuklagen. Nur weil ein anderer Arzt den Eindruck hat, dass an Stelle der vorgenommenen, an und für sich korrekten Operation vielleicht ein anderer Eingriff eher zum Erfolg geführt hätte, kann nicht von einem Kunstfehler und dementsprechend einem Haftpflichtanspruch ausgegangen werden.

\section{Unterläuft bei der Behandlung ein Fehler, soll} der Arzt unverzüglich Rücksprache mit seiner Haftpflichtversicherung nehmen.

Im Fokus der Arzthaftpflichtproblematik steht heute die Aufklärungspflicht. Der Patient sollte vom Operateur persönlich in einem Gespräch - nicht bloss durch Aushändigung eines Informationsblattes - ausführlich über die Risiken der vorgeschlagenen Behandlung und über alternative Behandlungsmöglichkeiten aufgeklärt werden. Zu Beweiszwecken ist ein schriftliches Aufklärungsprotokoll zu erstellen, das der Patient nach dem Gespräch unterschreibt. Unterläuft bei der Behandlung ein Fehler, soll der Arzt unverzüglich Rücksprache mit seiner Haftpflichtversicherung nehmen und mit dem Patienten den Fehler und die Möglichkeiten zu dessen Behebung diskutieren. Den Fehler $\mathrm{zu}$ verschleiern, den Patienten selber für das aufgetretene Problem verantwortlich zu machen oder die Diskussion zu verweigern, verhärtet die Fronten und vergrössert die Gefahr, dass es zu einer langwierigen und für alle Beteiligten zermürbenden gerichtlichen Auseinandersetzung kommt.

Der vorliegende Beitrag wurde in Zusammenarbeit mit RA Dr. iur. Cristina Schiavi, Küsnacht, verfasst. 